\title{
Antibiotic overuse: managing uncertainty and mitigating against overtreatment
}

\author{
Carolyn Tarrant (D) , ${ }^{1}$ Eva M Krockow ${ }^{2}$
}

${ }^{1}$ Department of Health Sciences, University of Leicester, Leicester, UK

${ }^{2}$ Department of Neuroscience, Psychology and Behaviour, University of Leicester, Leicester, UK

\section{Correspondence to} Professor Carolyn Tarrant, Department of Health Sciences, University of Leicester, Leicester, LE1 7RH, UK; ccp3@|e.ac.uk

Accepted 3 July 2021

\section{SLinked}

http://dx.doi.org/10.1136/ bmjgs-2020-012479

\section{Check for updates}

(c) Author(s) (or their employer(s)) 2021. No commercial re-use. See rights and permissions. Published by BMJ.

To cite: Tarrant $C$ Krockow EM. BMJ Qual Saf Epub ahead of print: [please include Day Month Year]. doi:10.1136/

bmjgs-2021-013615

\section{DIAGNOSTIC UNCERTAINTY DRIVES ANTIBIOTIC OVERUSE}

Antibiotic overuse contributes to the growing threat of antimicrobial resistance (AMR). Despite local, national and global initiatives to target the problem, reducing overuse is challenging. Levels of antibiotic prescribing in hospitals in the UK are still rising year on year. ${ }^{1}$ Pandolfo et al's ${ }^{2}$ study in this issue of BMJ Quality of Safety provides new insights into this problem by studying clinician decisionmaking about antibiotic prescribing in intensive care units, using a framework from psychology: the Necessity Concerns Framework. They explored how clinicians balanced the perceived necessity of antibiotics to protect the patient from deteriorating or dying from severe infection, against concerns about the actual or potential adverse consequences (including toxicity and the longer-term threat of AMR). Their findings highlight that clinical uncertainty is a complicating factor in this balancing act. When clinicians were unsure about the underlying cause of symptoms, they preferred to 'err on the side of caution' and give the patient antibiotics just to be safe.

Research into antibiotic prescribing across a range of healthcare settings has previously identified diagnostic uncertainty as a driving factor for overuse. ${ }^{3}$ Broom et al's ${ }^{4}$ qualitative study of pulmonary clinicians found that uncertainty drove reliance on antibiotics for hospital patients with respiratory symptoms. Aspects of uncertainty that played into these decisions included difficulty distinguishing between different possible diagnoses that could explain the patient's presenting symptoms and between bacterial and viral infection. When faced with a patient with a suspected infection, clinicians have a number of options. They can choose to order microbiology testing to inform their decision-making, or delay or withhold antibiotics and monitor the patient to see if symptoms resolve. But a common response to uncertainty about a suspected infection is to take action, by prescribing antibiotics 'just in case', to protect the patient from the immediate risk of deterioration. ${ }^{5}$ Reliance on antibiotics under conditions of uncertainty is widely reinforced by social norms in the local settings and among colleagues, and perceived patient expectations for active treatment. $^{67}$

Pandolfo et $a l^{2}$ look primarily to improved microbiology testing as a way of reducing uncertainty and avoiding antibiotic overuse. Testing, however, does not necessarily provide certainty. Broom et $a l^{4}$ also point to uncertainties arising from difficulties in interpreting the test results, in terms of distinguishing between the presence of a pathogen in a sample and colonisation causing infective symptoms. While improved testing can help inform decision-making, uncertainty can never be fully eliminated from the medical decision-making process. Rather than simply looking to technical solutions to try to remove uncertainty, efforts to reduce antibiotic overuse need to acknowledge uncertainty and identify ways of reducing reliance on antibiotics as a quick solution for managing uncertainty.

\section{FEATURES OF DECISION-MAKING UNDER UNCERTAINTY}

The reliance on active treatment as a response to uncertainty is grounded in fundamental features of human decisionmaking that have proved adaptive over millennia. Clinicians routinely have to make decisions under time pressure. Under these conditions, mental shortcuts (or heuristics) are effective at reducing 
cognitive load, and make decision-making more efficient when the right course of action is not immediately clear. But the use of heuristics can introduce decision errors resulting from cognitive biases. Some of these biases have particular relevance for antibiotic decision-making, ${ }^{8}$ including 'action bias', ${ }^{9}$ which refers to people's general preference for action over inaction even if the action is likely to result in poorer outcomes. Action bias is most commonly attributed to widespread social norms that interpret active choices as effortful and valuable, while labelling non-active choices as lazy alternatives that require little independent thought. Action bias is likely to increase if a decision-maker has had negative experiences in the past. Research shows that regret is higher for acts of omission than for those of commission. In other words, after negative decision outcomes, people experience higher levels of regret associated with actions they failed to take. ${ }^{10}$ In the context of antibiotic decision-making this means that negative patient outcomes following non-prescribing are likely to produce disproportionately large levels of regret. Doctors who previously chose an approach of watchful waiting for a patient with suspected infection, over immediate antibiotic prescribing, may experience emotionally painful regret associated with that decision. It is the anticipation of further regret that ultimately discourages similar, inactive choices in the future.

Risk aversion is another cognitive bias that drives decision-making under uncertainty. Risk aversion involves a preference for a sure or certain outcome over uncertainty, even if there are more potential gains to be had from tolerating uncertainty. ${ }^{11}$ Prescribing antibiotics 'just in case' is a risk-averse decision. In contrast, a decision not to prescribe, for the benefit of avoiding overtreatment and the long-term consequences of AMR, requires a clinician to be more tolerant of uncertainty and less risk-averse. Being comfortable with uncertainty comes with experience; evidence suggests that more experienced clinicians are better able to tolerate uncertainty and to make decisions that are less risk-averse. ${ }^{12}$

An additional important feature of clinical decisionmaking is that clinicians are making treatment recommendations and decisions for their patients; people think differently about risks and potential outcomes when making decisions for others. Preferences for risk-reducing, active, treatments are higher when people make treatment decisions for others as opposed to themselves. ${ }^{13}$ And evidence suggests that clinicians recommend treatments for their patients different from those they would choose for themselves-prioritising survival rates for their patients over other outcomes such as the risk of adverse side effects. ${ }^{14}$

In summary, there are fundamental human tendencies towards action in the face of uncertainty, a preference for certainty even at a cost and a desire to protect one's own patients from the risk of serious harm (figure 1). These tendencies underpin a reliance on active treatment, such as antibiotic prescribing, as a way of managing uncertainty. Psychosocial strategies that could have value in mitigating against antibiotic overuse as a response to uncertainty are outlined in figure 1 and described below. These include strategic
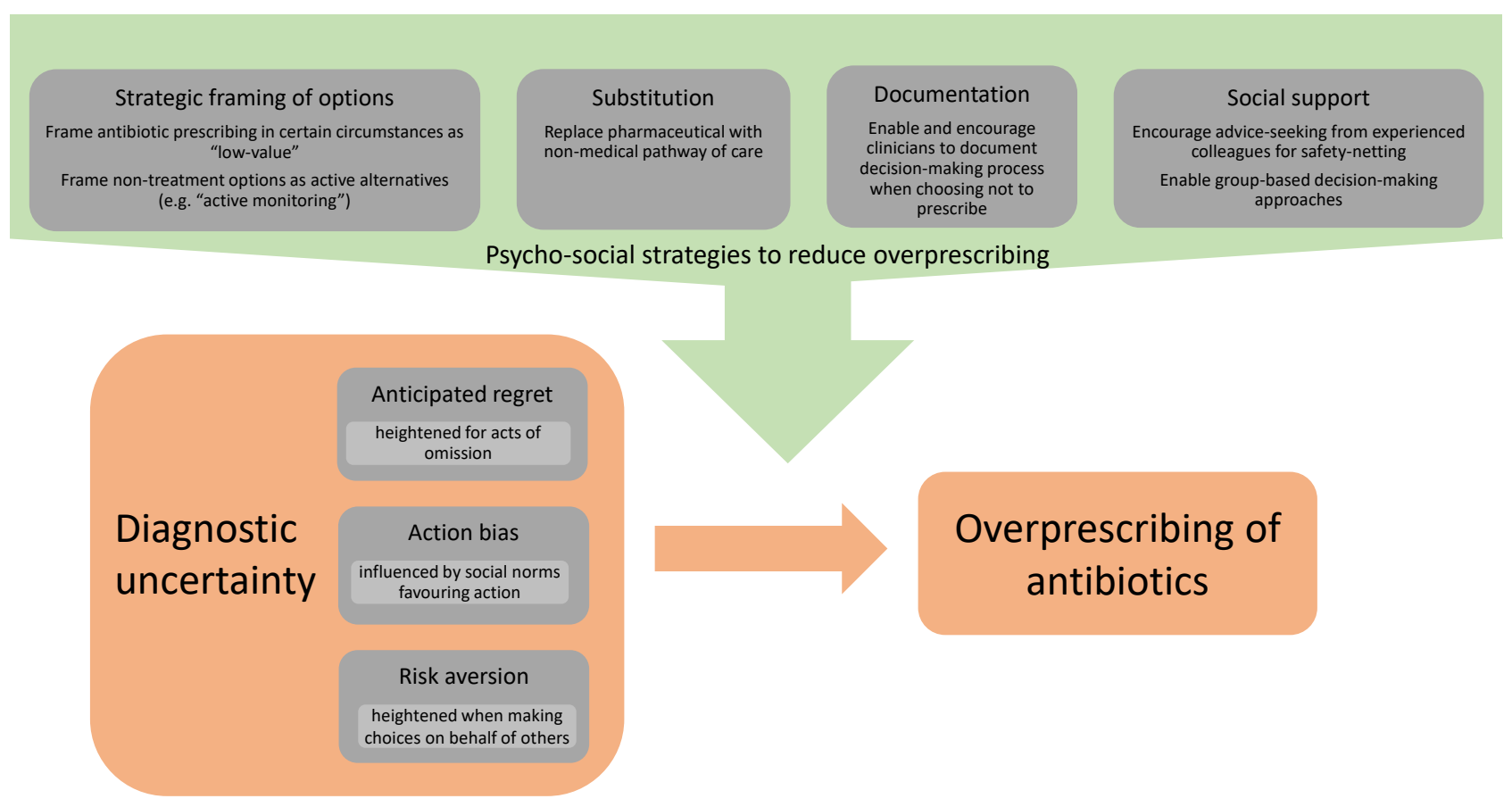

Figure 1 Diagnostic uncertainty and antibiotic overprescribing 
framing of options, substitution, documentation of decision-making processes and social support.

\section{HOW CAN WE REDUCE ANTIBIOTIC OVERUSE?}

Avoiding overtreatment is a common problem in medicine and requires clinicians to refrain from action, by not initiating tests or treatment when, on balance, these are not in the best interests of the patient or incur unnecessary costs (including costs to society). Efforts to reduce treatment overuse have used strategic framing $^{15}$ to label certain treatments as 'low value' forms of care. Specific directives, such as those in the Choosing Wisely campaign, ${ }^{16}$ provide guidelines to support avoidance of certain forms of treatment under certain circumstances. The campaign's 'do not do' directives encourage the avoidance of active treatment where it would be of low value or potentially cause patient harm. Drawing on this approach, formalising antibiotic prescribing guidelines to clearly outline the conditions under which antibiotic prescribing is likely to be of low value could play a role in supporting reductions in antibiotic overuse. Such guidelines and directives can encourage and legitimise decisions to avoid active treatment, but can be difficult to implement in practice: evidence about treatment value at population level may be seen as uninformative for individual-level decisions. ${ }^{17}$ Here, shared decision-making can play an important role. For example, in cancer treatment decisions, conversations supported by decision tools can help in assessing the value of treatment for an individual patient and can enable discussion about goals of care and the appropriateness of active (or more aggressive) intervention. ${ }^{18}$ In the case of antibiotic overuse, shared decision-making with patients or their relatives (when feasible) can provide an opportunity for a balanced discussion of uncertainties, risk and preferences. ${ }^{19}$ Shared decision-making approaches can also play a role in addressing clinicians' tendencies towards more risk-averse approaches to treatment when making decisions for others. Judgements about the value of antibiotic use are complicated, however, by tensions between the potential value of an antibiotic to an individual patient and the potential harm to society as a whole through AMR. ${ }^{5}$ The value to identifiable patients is likely to take precedence over agent-neutral harms-harms that are incurred by non-identifiable members of society. ${ }^{20}$ This holds particularly true in clinical cases characterised by extreme urgency (eg, patients presenting with symptoms of sepsis), where a sense of moral obligation to rescue the patient may be evoked (the so-called rule of rescue), ${ }^{21}$ outweighing any other considerations. The role of guidelines and shared decision-making in resolving these tensions is limited, as decision-making requires balancing of individual and collective risks and moral reasoning. ${ }^{22}$

A more promising line of enquiry might be to explore the strategic reframing of alternatives to active treatment. A choice not to prescribe an antibiotic could present a threat to the doctor-patient relationship if a patient views this as failure to provide necessary treatment and feels the clinician has not responded to their need for care. This issue is evident in other areas of medicine and has received particular attention in relation to end of life care, where efforts to reduce overtreatment to avoid patient harm have been problematic when interpreted as 'not doing'. Perkins and Fritz ${ }^{23}$ describe the reluctance of clinicians to discuss do not attempt resuscitation (DNAR) orders with patients and relatives because of a misconception that this equates with not providing any treatment. They argue for the need to frame these discussions in terms of goals of care and alternative actions, and to rename DNAR orders as 'Emergency Care Treatment Plans'. Similarly, in the field of cancer care, non-treatment choices that are intended to reduce the harm from overtreatment are deliberately framed using active language. In the management of certain cancers including prostate and colorectal, the terms 'active surveillance' or 'active monitoring' are used to describe a period of intensive monitoring as an alternative to passive terms like 'watchful waiting. ${ }^{24}$ Framing a non-medical approach to management of symptoms as an active alternative to antibiotic prescribing potentially mitigates against the impact of action bias on treatment decisions.

In a similar vein, Helfrich $e t a l^{25}$ draw on theories of cognitive processing to argue for the role of substitution in de-implementing low value or non-evidence-based practices. In the field of de-implementation, substitution involves replacing an undesired behaviour or practice with an alternative (eg, radiography replaced by CT). ${ }^{26}$ Substitution is more effective as a behaviour change strategy than simply asking people to stop an undesired behaviour, or to 'do nothing., ${ }^{27} 28$ This type of strategy avoids cognitively demanding processes, such as clinicians having to consciously reflect on their choices, or to unlearn and relearn new practices. Instead, it provides an easy alternative for action in line with the limitations of quick, heuristic decision-making approaches that clinicians use in reality. Drawing on this approach, there may be value in reframing antibiotic prescribing in terms of a choice between pharmaceutical and non-medical pathways of care for suspected infection. In this context, nonmedical forms of care such as the administration of fluids and enhanced monitoring would form an alternative, goal-directed, treatment pathway.

The clinicians interviewed in Pandolfo et al's study argued that they had to 'be brave' to withhold antibiotics, reflecting another key concern: the defensibility of decisions not to prescribe. Fear of censure from colleagues and reputational damage, and risk of litigation for failing to treat, have been identified as driving factors in antibiotic overuse. ${ }^{29}{ }^{30}$ Hedging-type defensive medicineexcessive use of tests and treatment as a defence against patient complaints, accusations of negligence and legal challenge-is reported as a common practice and a barrier to reducing overuse of medicine. ${ }^{31} \mathrm{~A}$ significant problem 
is that the process of decision-making about not taking action often remains undocumented. In the case of decisions about antibiotic use, this means that a choice not to prescribe antibiotics could be interpreted by others as an act of passive omission-a mistake or error-rather than active commission based on reasoned judgement. Enabling and encouraging clinicians to document the decisionmaking process behind non-prescribing, including documentation of patient involvement in discussions about value and goals of care, would provide a visible paper record: giving protection for clinicians from accusation of carelessness or neglect. ${ }^{32}$ Designing paperwork to allow documentation of uncertainty and of the evolving understanding of a patient's condition could enable clinicians to feel more comfortable with uncertainty and avoid the drive to establish certainty and take action. As Ries and Jansen $^{31}$ identify from their review of empirical research on defensive medicine, such individual-level approaches need to be accompanied by organisational-level culture change. In relation to antibiotic use, this would mean addressing the impact of initiatives and targets that disincentivise tolerance of uncertainty and encourage defensive medicine. $^{33}$

Although antibiotic decision-making is commonly understood as an individual behaviour, clinicians often consult with colleagues or expert advisors (such as microbiology, pharmacy or infectious diseases consultants) to inform their decision-making. For inexperienced clinicians, social support, through expert advice and senior review to provide safety-netting of their treatment decisions, can help them feel safer tolerating uncertainty. Role modelling by senior clinicians of how to deal with uncertainty has also been identified as a strategy for educating and socialising new doctors in ways of managing uncertainty. ${ }^{12}$ Research into group decision-making provides a further intriguing option for supporting decision-making about infection management under uncertainty. Evidence has shown that randomly created groups of three or more medical decision-makers overprescribe antibiotics less frequently than individual prescribers. ${ }^{34}$ As well as improving the outcomes of decisions made under uncertainty, the social support of a team is likely to lessen feelings of personal responsibility and risk susceptibility, reduce fear of litigation, and therefore lighten the difficult burden of uncertainty.

\section{CONCLUSIONS}

Pandolfo et al' $\mathrm{s}^{2}$ study provides more evidence that clinical uncertainty drives antibiotic overuse. The role of uncertainty in antibiotic overuse is important but frequently overlooked. Antimicrobial stewardship approaches that are predicated on auditing and 'correcting' prescribing behaviour fail to take into account the uncertainties around diagnosis and management of infection and how antibiotic overuse can result from the clinicians' drive to reduce uncertainty and protect the patients in their care from harm. Equally, technical solutions such as improved diagnostic tests can never fully resolve uncertainties.
Recognising that uncertainty is an inherent aspect of medicine and considering how best to support clinicians to avoid reliance on antibiotics as a remedy for uncertainty should be the foundation for future efforts to mitigate against antibiotic overuse in clinical practice.

Twitter Carolyn Tarrant @ carolynctarrant

Acknowledgements The authors are grateful for the helpful suggestions and comments by Perla Marang-van de Mheen during the preparation of this article.

Contributors CT and EMK conceived the paper, read and modified subsequent drafts, and approved the final version.

Funding The authors have not declared a specific grant for this research from any funding agency in the public, commercial or not-for-profit sectors.

Competing interests None declared.

Patient consent for publication Not required.

Provenance and peer review Commissioned; internally peer reviewed.

\section{ORCID iD}

Carolyn Tarrant http://orcid.org/0000-0001-7356-5342

\section{REFERENCES}

1 Public Health England. English surveillance programme for antimicrobial utilisation and resistance (ESPAUR); 2020. https://assets.publishing.service.gov.uk/government/uploads/ system/uploads/attachment_data/file/936199/ESPAUR_Report_ 2019-20.pdf

2 Pandolfo AM, Horne R, Jani Y, et al. Understanding decisions about antibiotic prescribing in ICU: an application of the necessity concerns framework. BMJ Qual Saf 2021. doi:10.1136/bmjqs-2020-012479. [Epub ahead of print: 07 Jun 2021].

3 Stefan MS, Spitzer KA, Zulfiqar S, et al. Uncertainty as a critical determinant of antibiotic prescribing in patients with an asthma exacerbation: a qualitative study. J Asthma 2020:1-16.

4 Broom JK, Broom AF, Kirby ER, et al. Clinical and social barriers to antimicrobial stewardship in pulmonary medicine: a qualitative study. Am J Infect Control 2017;45:911-6.

5 Krockow EM, Colman AM, Chattoe-Brown E, et al. Balancing the risks to individual and society: a systematic review and synthesis of qualitative research on antibiotic prescribing behaviour in hospitals. J Hosp Infect 2019;101:1-18.

6 McDonnell Norms Group. Antibiotic overuse: the influence of social norms. J Am Coll Surg 2008;207:265-75.

7 Cabral C, Lucas PJ, Ingram J, et al. "It's safer to ..." parent consulting and clinician antibiotic prescribing decisions for children with respiratory tract infections: An analysis across four qualitative studies. Soc Sci Med 2015;136-137:156-64.

8 Langford BJ, Daneman N, Leung V, et al. Cognitive bias: how understanding its impact on antibiotic prescribing decisions can help advance antimicrobial stewardship. JAC Antimicrob Resist 2020;2:dlaa107.

9 Ayanian JZ, Berwick DM. Do physicians have a bias toward action? A classic study revisited. Med Decis Making 1991;11:154-8.

10 Zeelenberg M, van den Bos K, van Dijk E, et al. The inaction effect in the psychology of regret. J Pers Soc Psychol 2002;82:314-27.

11 Werner J, Aversion R. The new palgrave dictionary of economics. London: Palgrave Macmillan, 2018. 
12 Lawton R, Robinson O, Harrison R, et al. Are more experienced clinicians better able to tolerate uncertainty and manage risks? A vignette study of doctors in three NHS emergency departments in England. BMJ Qual Saf 2019;28:382-8.

13 Ubel PA, Angott AM, Zikmund-Fisher BJ. Physicians recommend different treatments for patients than they would choose for themselves. Arch Intern Med 2011;171:630-4.

14 Zikmund-Fisher BJ, Sarr B, Fagerlin A, et al. A matter of perspective: choosing for others differs from choosing for yourself in making treatment decisions. J Gen Intern Med 2006;21:618-22.

15 Entman RM. Framing: toward clarification of a fractured paradigm. J Commun 1993;43:51-8.

16 Levinson W, Kallewaard M, Bhatia RS, et al. 'Choosing wisely': a growing international campaign. BMJ Qual Saf 2015;24:167-74.

17 Smith ME, Vitous CA, Hughes TM, et al. Barriers and facilitators to de-implementation of the Choosing Wisely ${ }^{\circledR}$ guidelines for low-value breast cancer surgery. Ann Surg Oncol 2020;27:1-11.

18 Lifford KJ, Edwards A, Burton M, et al. Efficient development and usability testing of decision support interventions for older women with breast cancer. Patient Prefer Adherence 2019;13:131-43.

19 Butler CC, Kinnersley P, Prout H, et al. Antibiotics and shared decision-making in primary care. J Antimicrob Chemother 2001;48:435-40.

20 McKie J, Richardson J. The rule of rescue. Soc Sci Med 2003;56:2407-19.

21 Krockow EM, Tarrant C. The International dimensions of antimicrobial resistance: contextual factors shape distinct ethical challenges in South Africa, Sri Lanka and the United Kingdom. Bioethics 2019;33:756-65.

22 Tarrant C, Krockow EM, Nakkawita WMID, et al. Moral and contextual dimensions of "inappropriate" antibiotic prescribing in secondary care: A three-country interview study. Front Sociol 2020;5:7.

23 Perkins GD, Fritz Z. Time to change from do-not-resuscitate orders to emergency care treatment plans. JAMA Netw Open 2019;2:e195170-e70.
24 Bruinsma SM, Roobol MJ, Carroll PR, et al. Semantics in active surveillance for men with localized prostate cancer results of a modified Delphi consensus procedure. Nat Rev Urol 2017;14:312-22.

25 Helfrich CD, Rose AJ, Hartmann CW, et al. How the dual process model of human cognition can inform efforts to de-implement ineffective and harmful clinical practices: a preliminary model of unlearning and substitution. J Eval Clin Pract 2018;24:198-205.

26 van Bodegom-Vos L, Davidoff F, Marang-van de Mheen PJ. Implementation and de-implementation: two sides of the same coin? BMJ Qual Saf 2017;26:495-501.

27 Patey AM, Hurt CS, Grimshaw JM, et al. Changing behaviour 'more or less'-do theories of behaviour inform strategies for implementation and de-implementation? A critical interpretive synthesis. Implementation Sci 2018;13:1-13.

28 Michie S, Richardson M, Johnston M, et al. The behavior change technique taxonomy (V1) of 93 hierarchically clustered techniques: building an international consensus for the reporting of behavior change interventions. Ann Behav Med 2013;46:81-95.

29 Broom A, Kirby E, Gibson AF, et al. Myth, manners, and medical ritual: defensive medicine and the fetish of antibiotics. Qual Health Res 2017;27:1994-2005.

30 Tebano G, Dyar OJ, Beovic B, et al. Defensive medicine among antibiotic stewards: the International ESCMID AntibioLegalMap survey. J Antimicrob Chemother 2018;73:1989-96.

31 Ries NM, Jansen J. Physicians' views and experiences of defensive medicine: an international review of empirical research. Health Policy 2021;125:634-42.

32 Armstrong N. Overdiagnosis and overtreatment: a sociological perspective on tackling a contemporary healthcare issue. Sociol Health Illn 2021;43:58-64.

33 Fitzpatrick F, Tarrant C, Hamilton V, et al. Sepsis and antimicrobial stewardship: two sides of the same coin. BMJ Qual Saf 2019;28:758-61.

34 Krockow EM, Kurvers RHJM, Herzog SM, et al. Harnessing the wisdom of crowds can improve guideline compliance of antibiotic prescribers and support antimicrobial stewardship. Sci Rep 2020;10:1-12. 